\title{
Random Fibonacci Sequences and Capacity/Power Scaling in Cooperative Multihop Networks
}

\author{
David E. Simmons, and Justin P. Coon \\ Department of Engineering Science \\ University of Oxford \\ Oxford, UK, OX13PJ \\ Email: \{david.simmons, justin.coon\}@eng.ox.ac.uk
}

\begin{abstract}
In this paper, we analyze capacity and power scaling in multihop cooperative AF relay networks. An analytical framework for this task is developed by drawing a correspondence between random Fibonacci sequences and the end-to-end multihop system model. It turns out, the exponential growth rate of these interesting sequences can be employed to establish scaling laws, from which we conclude that it is possible to construct multihop cooperative AF networks that simultaneously avoid 1) exponential capacity decay and 2) exponential transmit power growth across the network. This is done by ensuring the network's Lyapunov exponent (a key observable studied in random dynamical system theory) is zero, which can be achieved by appropriately selecting the amplification factors at each of the relay nodes. Our results apply to both fixed-gain and variable-gain relaying. To conclude our work, we demonstrate the presented theory through numerical simulations.

Index Terms-Relay network, amplify-and-forward, capacity, random dynamical system, RDS, Lyapunov exponent, scaling.
\end{abstract}

\section{INTRODUCTION}

In recent years there has been a concerted effort to understand the behavior of multihop relay systems. For the linear multihop scenario [1], [2] it is known that the capacity of such networks is achieved by employing decode-and-forward (DF) [3] relaying. This can be seen by considering the max-flow min-cut theorem. However, if we consider nonlinear topologies (i.e., internode connections extending beyond a single hop, see Fig. 1) the optimal (in the capacity sense) forwarding strategy is still unknown [4]. Consequently, for these topologies we are limited to studying the fundamental limits of performance when certain specific relaying strategies are employed. Forwarding strategies that are of particular interest to the literature are DF and amplify-and-forward (AF) [3]. DF relays are attractive because (provided the channel capacity permits) they can successfully remove noise without propagating it onto the next node in the network. However, when larger numbers of nodes are deployed, DF-based protocols may result in prohibitive latency/complexity because of the decoding process that takes place at each relay. AF protocols become interesting at this point since they can be employed to yield low complexity and/or low latency solutions. Under certain scenarios, they also have the potential to offer greater diversity when compared to DF schemes [3].

It is known that the analysis of DF networks [3] is made easier by the fact that a local view can often be taken - i.e., the transmission is "reset" at each relay node, and thus sequential hops can, to a certain extent, be treated independently. This is not the case for AF networks, which must be observed globally in the general case since the end-toend transmission is affected by a composition of mappings, one for each hop. Consequently, the study of multihop AF networks often requires more advanced mathematical tools, e.g., random matrix theory [5] or RDS theory [6]. In [7], [8], multihop AF networks are studied through the formalism of random matrix theory, while [1], [2] studies these networks through the formalism of RDS theory.

The aim of this paper is to show that much of the analysis performed in [1], which focused on studying AF linear multihop networks through the formalism of RDS theory (and Lyapunov exponents, key observable studied in RDS theory), can be extended to a certain class of AF network that possesses the nonlinear topology depicted in Fig. 1. This is done by considering the exponential growth rate of random Fibonacci sequences of the form

$$
I_{n}=\eta_{n-1, n} I_{n-1}+\eta_{n-2, n} I_{n-2},
$$

where $\left\{\eta_{i, j}\right\}$ is a set of strictly positive i.i.d. random variables with continuous distribution. In this work we demonstrate that for a cooperative AF relay network:

1) The rate at which the end-to-end capacity will decay can be determined by the exponential decay rate of certain random Fibonacci sequences.

2) The rate at which the $n$th node transmit power will grow can be determined by the exponential growth rate of certain random Fibonacci sequences.

An immediate consequence of our result is that exponential capacity decay and exponential transmit power growth can be simultaneously avoided across linear-like networks, provided the network designer configures the relays' gains such that the network's Lyapunov exponent is equal to zero. These results will have practical implications for communication along delay intolerant networks such as, e.g., vehicular convoys or sensor along railways lines, etc.

\section{A. Notation, Definitions, and Layout}

In this work, logarithms are always base $e$ and matrices are always represented using uppercase boldface notation. $\log ^{+} x:=\max \{0, \log x\}$ and $\sigma_{i}(\mathbf{A})$ is used to denote the $i$ th 


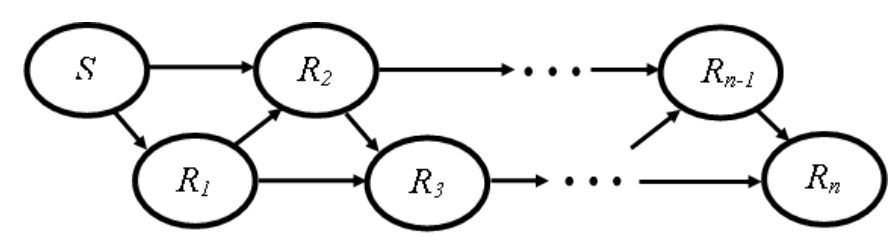

Fig. 1. Depiction of a cooperative relay network. Signals from the $i$ th node are assumed to travel as far as the $i+2$ th node.

ordered singular value of the matrix $\mathbf{A}$, where $\sigma_{i}(\mathbf{A}) \geq \sigma_{j}(\mathbf{A})$ implies $i \leq j$. Similar to notation used in [1], for a strictly positive random variable $f(n)$ depending on $n$, and some $h(n)=|o(n)|$

$f(n)=O_{\mathbb{P}}(g(n)) \Rightarrow \lim _{n \rightarrow \infty} \mathbb{P}\left[f(n) \leq g(n) e^{h(n)}\right]=1$.

$f(n)=\Omega_{\mathbb{P}}(g(n)) \Rightarrow \lim _{n \rightarrow \infty} \mathbb{P}\left[f(n) \geq g(n) e^{-h(n)}\right]=1$

$f(n)=\Theta_{\mathbb{P}}(g(n))$ if $f(n)=O_{\mathbb{P}}(g(n))$ and $f(n)=\Omega_{\mathbb{P}}(g(n))$.

The remainder of this paper is as follows. Section II-A discusses background information relating to RDS theory and Lyapunov exponents. Section III presents the system model of the communication network considered in this paper. Section IV presents the fundamental result of this paper, while section $\mathrm{V}$ present some numerical examples to verify our theoretical analysis. Section VI concludes the paper.

\section{RANDOM SEQUENCES AND DynamicAl Systems}

With $F_{1}=F_{2}=1$, the $n$th Fibonacci number is defined to be $F_{n}=F_{n-1}+F_{n-2}$, and is named after the Italian mathematician Leonardo Fibonacci [9]. These numbers frequently arise in nature [10]; e.g., the leaf arrangement on a stem, the spiral number in the floret of a sunflower, and the ancestry code of a male bee [11]. Fibonacci numbers are also connected to the golden ratio

$$
\phi:=\frac{1+\sqrt{5}}{2},
$$

which has been used to proportion the work of artists and architects throughout history [12]. In particular, it is well known that

$$
\lim _{n \rightarrow \infty}\left(F_{n} / F_{n-1}\right)=\phi .
$$

Random Fibonacci sequences were first introduced in 2000 [13], where it was shown that, with $x_{n}$ defined by

$$
x_{n+1}= \pm x_{n} \pm x_{n-1}
$$

where the signs are chosen independently and with equal probability, the limit

$$
\lim _{n \rightarrow \infty}\left|x_{n}\right|^{\frac{1}{n}} \stackrel{a . s .}{=} C
$$

exists almost surely (a.s.) with $C=1.13198824 \ldots$ (Viswanath's constant). This result can be framed within the context of random dynamical system (RDS) theory ${ }^{1}$ by writing (2) in matrix form as

$$
\left[\begin{array}{c}
x_{n-1} \\
x_{n}
\end{array}\right]=\prod_{i=2}^{n}\left[\begin{array}{cc}
0 & 1 \\
\pm 1 & \pm 1
\end{array}\right]\left[\begin{array}{l}
x_{0} \\
x_{1}
\end{array}\right]
$$

where, as with before, the signs are chosen independently and with uniform probability. We can then refer to the Lyapunov exponent of this RDS, which in this case is given by

$$
\lambda_{x}=\log C .
$$

The Lyapunov exponent is a key observable studied in RDS theory, and describes the exponential growth rate of the norm of a trajectory as it propagates through a phase space according to a particular RDS.

The random Fibonacci sequence was generalized to

$$
x_{n+1}= \pm x_{n} \pm \beta x_{n-1}
$$

$\beta>0$, and studied further in [14], where it was shown that there was a critical threshold $\beta^{\star}$ such that $\beta>\beta^{\star}$ would cause exponential growth in the sequence, while $\beta<\beta^{\star}$ would cause exponential decay. Further work was performed in [15], where the exponential growth rate of

$$
x_{n+1}=x_{n} \pm x_{n-1}
$$

was assessed under the assumption that + is chosen with probability $p$ and - with $1-p$. Other efforts to understand such sequences can be found in [16]-[18].

To the best of the authors' knowledge, there has been no such study of random Fibonacci sequences that take the form

$$
I_{n}=\eta_{n-1, n} I_{n-1}+\eta_{n-2, n} I_{n-2},
$$

where $\left\{\eta_{i, j}\right\}$ is a set of strictly positive i.i.d. random variables with continuous distribution where $\mathbb{E} \log \eta_{i, j}<\infty$,

$$
I_{1}:=\eta_{0,1} I_{0},
$$

and $I_{0}$ belongs to some bounded domain in $\mathbb{R}^{+}$. We call this type of sequence a continuous random Fibonacci sequence. One of the focuses of this paper is to motivate the study of such sequences through their application to problems in cooperative wireless communication networks.

\section{A. Dynamical Systems}

For the interested reader, let us briefly review RDSs and Lyapunov exponents (for a more comprehensive overview of RDS theory, the reader should refer to [6]). We consider an RDS to be a successive application of $d \times d(d \in \mathbb{N})$ complex random matrices on an appropriately dimensioned vector (the initial state $\left.X_{0} \in \mathbb{C}^{d}\right)$. Mathematically, the state of the RDS at time $n\left(X_{n} \in \mathbb{C}^{d}\right)$ can then be written as

$$
X_{n}=\mathbf{A}_{n} \cdots \mathbf{A}_{1} X_{0},
$$

\footnotetext{
${ }^{1}$ We will provide a brief review of RDS theory in section II-A.
} 
where, in general, we assume that $\mathbf{A}_{1}, \ldots, \mathbf{A}_{n}$ are independent and identically distributed up to an arbitrary positive scaling factor; i.e., $\exists \beta_{i}>0$ such that

$$
\mathbf{A}_{1} \stackrel{d}{=} \beta_{i} \mathbf{A}_{i}
$$

for all $i$. The RDS's Lyapunov exponent is given in the following definition.

Definition The Lyapunov exponent of the RDS given in (6), with initial state $X_{0}$, is defined to be

$$
\lambda_{\mathbf{A}}\left(X_{0}\right):=\lim _{n \rightarrow \infty} \frac{1}{n} \log \left\|\mathbf{A}_{n} \cdots \mathbf{A}_{1} X_{0}\right\| .
$$

To aid our understanding of the Lyapunov exponent, consider the following discussion. Suppose we wish to study the spectral properties of the RDS in (6), i.e.,

$$
\sigma_{i}\left(\mathbf{A}_{n} \cdots \mathbf{A}_{1}\right), \forall i
$$

as $n \rightarrow \infty$. One route is to exponentiate the logarithm of (8); i.e., write (8) as

$$
\sigma_{i}\left(\mathbf{A}_{n} \cdots \mathbf{A}_{1}\right)=e^{n \frac{1}{n} \log \sigma_{i}\left(\mathbf{A}_{n} \cdots \mathbf{A}_{1}\right)}
$$

and investigating the behavior of the term $\frac{1}{n} \log \sigma_{i}\left(\mathbf{A}_{n} \cdots \mathbf{A}_{1}\right)$ as $n$ grows large. In this manner, the exponential growth/decay rate of the system's spectrum can be observed. If $\left\{\mathbf{A}_{j}\right\}$ is a set of scalars (i.e., $d=1$ ), the law of large numbers can be employed to evaluate $\lim _{n \rightarrow \infty} \frac{1}{n} \log \sigma_{i}\left(\mathbf{A}_{n} \cdots \mathbf{A}_{1}\right)$; however, this is not the case for general $d$.

For general $d$, and under the condition that $\mathbb{E}\left[\log ^{+}\left\|\mathbf{A}_{1}\right\|\right]<$ $\infty$ and $\lim _{n \rightarrow \infty} 1 / n \sum_{i=1}^{n} \log ^{+}\left|\beta_{i}\right|<\infty$, the theorem of Furstenberg and Kesten [6] guarantees that the limit exists. This leads us to the following fact about Lyapunov exponents.

Fact 1: With the assumption that $\mathbb{E}\left[\log ^{+}\left\|\mathbf{A}_{1}\right\|\right]<\infty$ and $\lim _{n \rightarrow \infty} 1 / n \sum_{i=1}^{n} \log ^{+}\left|\beta_{i}\right|<\infty[6]$ :

A. The Furstenberg-Kesten theorem guarantees that $\forall i$

$$
\lambda_{\mathbf{A}, i}:=\lim _{n \rightarrow \infty} \frac{1}{n} \log \sigma_{i}\left(\mathbf{A}_{n} \cdots \mathbf{A}_{1}\right) \text { exists. }
$$

B. Through the multiplicative ergodic theorem,

$\lambda_{\mathbf{A}}: \mathbb{C}^{d} \mapsto\left\{\lambda_{\mathbf{A}, 1}, \ldots, \lambda_{\mathbf{A}, d}\right\}$ and $\lambda_{\mathbf{A}}(\cdot)$ is surjective.

\section{Cooperative Network System Model}

Consider a cooperative AF relay network with topology depicted in Fig. 1. The source (node $S$ ) transmits an information symbol $x \in \mathbb{C}$ drawn from a standard constellation (e.g., QAM) with some magnitude $I_{0}$ to the first and second relays (nodes $R_{1}$ and $R_{2}$ ). The first and second relays then, respectively, receive

$$
x_{01}=x_{0} h_{01}+v_{01} \quad \text { and } \quad x_{02}=x_{0} h_{02}+v_{02},
$$

where $h_{i j} \sim \mathcal{C N}\left(\mu_{i j}\right)$ denotes the channel coefficient between nodes $i$ and $j$, and $v_{i j} \sim \mathcal{C N}\left(n_{0}\right)$ denotes the noise received at node $j$ as it was listening to node $i$. These relays then correct the phase shifts introduced by the channels between themselves and the source to obtain

$$
\tilde{x}_{01}=x_{0}\left|h_{01}\right|+v_{01} \quad \text { and } \quad \tilde{x}_{02}=x_{0}\left|h_{02}\right|+v_{02} .
$$

Note, in (12) for brevity we have ignored the phase shift that should also be applied to the noise term because the resulting calculations are unchanged. In the next time-slot, the first relay amplifies and transmits $\tilde{x}_{01}$ to the second and third relays so that they receive

$$
x_{12}=h_{12} g_{1}\left(x_{0}\left|h_{01}\right|+v_{01}\right)+v_{12}
$$

and

$$
x_{13}=h_{13} g_{1}\left(x_{0}\left|h_{01}\right|+v_{01}\right)+v_{13},
$$

where $g_{i}$ is the amplification factor applied at node $i$. The second and third relays again correct the phase shift introduced by the channel to obtain

$$
\tilde{x}_{12}=\left|h_{12}\right| g_{1}\left(x_{0}\left|h_{01}\right|+v_{01}\right)+v_{12}
$$

and

$$
\tilde{x}_{13}=\left|h_{13}\right| g_{1}\left(x_{0}\left|h_{01}\right|+v_{01}\right)+v_{13} .
$$

The second relay then transmits

$$
\tilde{x}_{2}=g_{2}\left(\tilde{x}_{02}+\tilde{x}_{12}\right)
$$

to the third and fourth relays. This process continues, and in general the $i$ th relay:

1) separately performs phase alignment on the signals received from the $(i-1)$ th and $(i-2)$ th nodes;

2) sums the result, amplifies it, and transmits it to the $(i+1)$ th and $(i+2)$ th nodes.

Consequently, the magnitude (not the power, i.e., the squared magnitude) of the transmitted information signal, $I_{n}$, at the $n$th node can be obtained from the random Fibonacci sequence given in (4), where

$$
\eta_{i, j}:=\left|h_{i, j} g_{j}\right| .
$$

In this work, the gains are assumed to be arbitrary (e.g., they may be fixed (fixed-gain) or vary with the channel (variablegain)).

The information symbol transmitted at the $n$th node can be converted into the following matrix expression

$$
\left[\begin{array}{c}
I_{n-1} \\
I_{n}
\end{array}\right]=\prod_{i=2}^{n}\left[\begin{array}{cc}
0 & 1 \\
\eta_{i-2, i} & \eta_{i-1, i}
\end{array}\right]\left[\begin{array}{c}
I_{0} \\
\eta_{0,1} I_{0}
\end{array}\right],
$$

which is required so that we can leverage results from RDS theory, [6]. After averaging over the noise, the transmitted noise power at the $n$th node, $N_{n}^{2}$, can be obtained from the matrix system

$$
\left[\begin{array}{c}
N_{n-1}^{2} \\
N_{n}^{2} \\
1
\end{array}\right]=\prod_{i=2}^{n}\left[\begin{array}{ccc}
0 & 1 & 0 \\
\eta_{i-2, i}^{2} & \eta_{i-1, i}^{2} & n_{0} \\
0 & 0 & 1
\end{array}\right]\left[\begin{array}{c}
0 \\
n_{0} \\
1
\end{array}\right],
$$

so that the SNR at the $n$th node is given by

$$
\gamma_{n}:=\frac{I_{n}^{2}}{N_{n}^{2}},
$$

and the transmitted power is given by

$$
X_{n}^{2}=I_{n}^{2}+N_{n}^{2} .
$$

With (20), we can write the capacity of the network as [19]

$$
c_{n}=\log \left(1+\gamma_{n}\right) \text { nats/channel use. }
$$




\section{CAPACITY AND POWER SCALing LaWS For COOPERATIVE RELAY Networks}

$$
O=\zeta I+D
$$

In this section, we establish capacity and power scaling laws for the network described in section III. We begin with capacity in the following theorem.

Theorem 1: Let $\lambda$ be the upper Lyapunov exponent of the RDS described in (18). Then the following limit exists almost surely

$$
\lambda_{\gamma}:=\lim _{n \rightarrow \infty} \frac{1}{n} \log c_{n} \stackrel{\text { a.s. }}{=} \min \{0,2 \lambda\} .
$$

Thus, the capacity at the $n$th node is given by

$$
c_{n}=\Theta_{\mathbb{P}}\left(\exp \left(n \lambda_{\gamma}\right)\right) .
$$

Proof: The theorem follows from [1, Theorem 1], which shows that

$$
\lambda_{N}:=\lim _{n \rightarrow \infty} \frac{1}{n} \log N_{n}^{2},
$$

is given by

$$
\lambda_{N} \stackrel{a . s .}{=} \max \{0,2 \lambda\} .
$$

From (20) and (25), we obtain

$$
\begin{aligned}
\lim _{n \rightarrow \infty} \frac{1}{n} \log \gamma_{n} & =\lim _{n \rightarrow \infty} \frac{1}{n} \log I_{n}^{2}-\lim _{n \rightarrow \infty} \frac{1}{n} \log N_{n}^{2} \\
& =2 \lambda-\max \{0,2 \lambda\}=\min \{0,2 \lambda\} .
\end{aligned}
$$

The existence of these limits follows from the FurstenbergKesten theorem. From (22), (26) and [1, Fact 2.4] we have

$$
\mathbb{P}\left[\log \left(e^{n \lambda_{\gamma}-o(n)}+1\right) \leq c_{n} \leq \log \left(e^{n \lambda_{\gamma}+o(n)}+1\right)\right] \rightarrow 1,
$$

which gives

$$
\begin{aligned}
& \mathbb{P}\left[e^{n \lambda_{\gamma}-o(n)}+O\left(e^{2 n \lambda_{\gamma}}\right) \leq\right. \\
&\left.c_{n} \leq e^{n \lambda_{\gamma}+o(n)}+O\left(e^{2 n \lambda_{\gamma}}\right)\right] \rightarrow 1 \\
& \Rightarrow \mathbb{P}\left[e^{n \lambda_{\gamma}-o(n)} O(1) \leq c_{n} \leq e^{n \lambda_{\gamma}+o(n)} O(1)\right] \rightarrow 1 \\
& \Rightarrow \mathbb{P}\left[e^{n \lambda_{\gamma}-o(n)} \leq c_{n} \leq e^{n \lambda_{\gamma}+o(n)}\right] \rightarrow 1
\end{aligned}
$$

where (for $\lambda_{\gamma}<0$ ) the first line follows from the Taylor expansion of $\log (1+x)$ about $x=0$ and the second line follows by factoring $e^{n \lambda_{\gamma} \pm o(n)}$ from the left and right sides of the first line, respectively. From the last line, the stated result then follows from the definition of $\Theta_{\mathbb{P}}(\cdot)$. For $\lambda_{\gamma}=0$, the RHS of the first line becomes $o(n) \leq e^{o(n)}$, so the result still holds.

In the next lemma, we will determine the power scaling properties of the network. With this, we will be able to establish the relationship between the decay of the capacity across the network and the growth in its transmit power. In particular, we will conclude that it is possible to construct cooperative AF networks that avoid both exponential capacity decay and exponential transmit power growth.
Lemma 1: Let $\lambda$ be the upper Lyapunov exponent of the RDS described in (18). Then the following limit exists almost surely

$$
\lambda_{X}:=\lim _{n \rightarrow \infty} \frac{1}{n} \log X_{n}^{2} \stackrel{\text { a.s. }}{=} \min \{0,2 \lambda\} .
$$

Thus, the transmit power at the $n$th node is given by

$$
X_{n}^{2}=\Theta_{\mathbb{P}}\left(\exp \left(n \lambda_{X}\right)\right) .
$$

Proof: From (21), it is clear that

$$
\frac{1}{n} \log N_{n}^{2} \leq \frac{1}{n} \log X_{n}^{2} \leq \frac{1}{n} \log 2 \max \left\{I_{n}^{2}, N_{n}^{2}\right\} .
$$

By considering (25), we can take the limit of (28) to obtain

$$
\lim _{n \rightarrow \infty} \frac{1}{n} \log X_{n}^{2}=\max \{0,2 \lambda\} .
$$

The stated result then follows from [1, Fact 2.4].

Theorem 1 and Lemma 1 demonstrate that it is possible to construct a cooperative AF network that simultaneously avoids exponential capacity decay and exponential transmit power growth. To achieve this, the network must be constructed such that its Lyapunov exponent is zero; i.e., $\lambda=0$. The network designers can do this by appropriately selecting the relay nodes' amplification factors.

\section{Discussion AND Numerical Results}

We begin our discussion by providing a graphical depiction (Fig. 2) of the relationship between the average per-hop total channel gain (i.e., $\mathbb{E}\left[\left|g_{i}^{2} h_{i-1, i}\right|\right]$ ) and the network's Lyapunov exponent $\lambda$ (see Theorem 1). In this figure, the Lyapunov exponent is calculated numerically by considering the finite Lyapunov exponent of the matrix system shown in (18), which is defined to be

$$
\lambda_{n}:=\frac{1}{n} \log \left|\prod_{i=2}^{n}\left[\begin{array}{cc}
0 & 1 \\
\eta_{i-2, i} & \eta_{i-1, i}
\end{array}\right]\left[\begin{array}{c}
I_{0} \\
\eta_{0,1} I_{0}
\end{array}\right]\right|,
$$

where

$$
\lim _{n \rightarrow \infty} \lambda_{n}=\lambda,
$$

and evaluating $\lambda_{n}$ at $n=100,000$. We assume that each of the relays applies a unit gain $g_{i}=1$ and that the channel coefficients are i.i.d. with $\mathbb{E}\left[\left|h_{i, j}\right|^{2}\right]=\mu$. From this figure, we can see that the Lyapunov exponent crosses from negative to positive at the point $\mathbb{E}\left[\left|g_{j} h_{i, j}\right|^{2}\right] \approx 0.35$. In the following discussion, we will frequently refer to this figure and in particular the critical point at which $\lambda=0$. For extra clarity, Fig. 3 shows how the finite Lyapunov exponent (solid lines) converges to the actual Lyapunov exponent ${ }^{2}$ (dashed lines) with the number of hops in the network. From this figure, we see that it is not until the network is of $\approx 100$ nodes in length before $\lambda_{n}$ begins to trend towards $\lambda$.

With reference to Fig. 4, we will now demonstrate Theorem 1. In this figure, a particular realization of capacity (22) is plotted (solid lines) as a function of network size. This figure also contains plots of $\exp \left(n \lambda_{\gamma}\right)$ (see (23)) (dashed

\footnotetext{
${ }^{2}$ Here, the dashed lines are calculated by considering large $n=100,000$.
} 


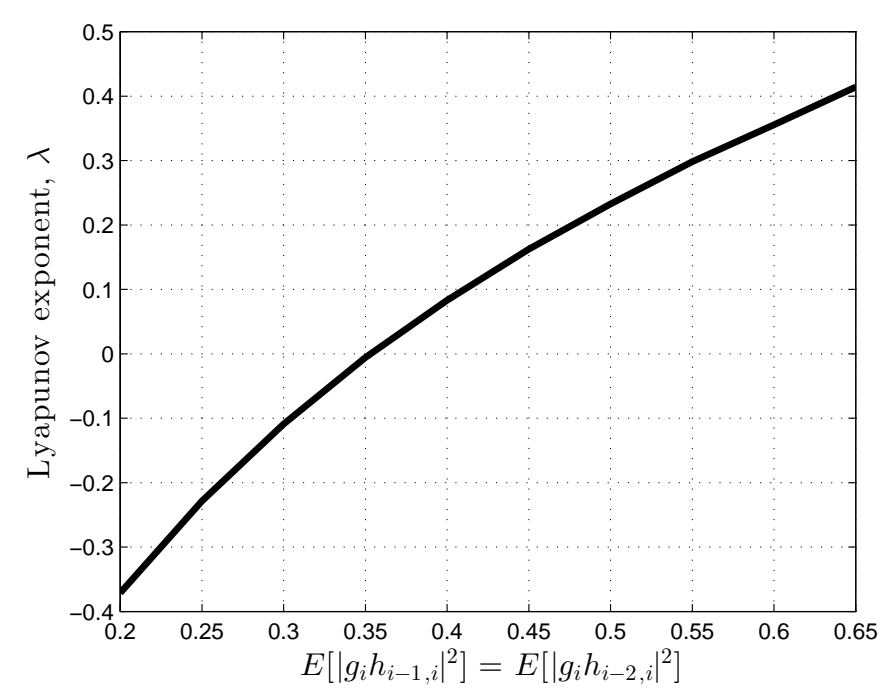

Fig. 2. Figure showing numerical calculation of the network's Lyapunov exponent (see Theorem 1) as a function of the average per-hop total channel gain. The relays are assumed to apply a unit gain and the channel coefficients between each node are assumed to be i.i.d.

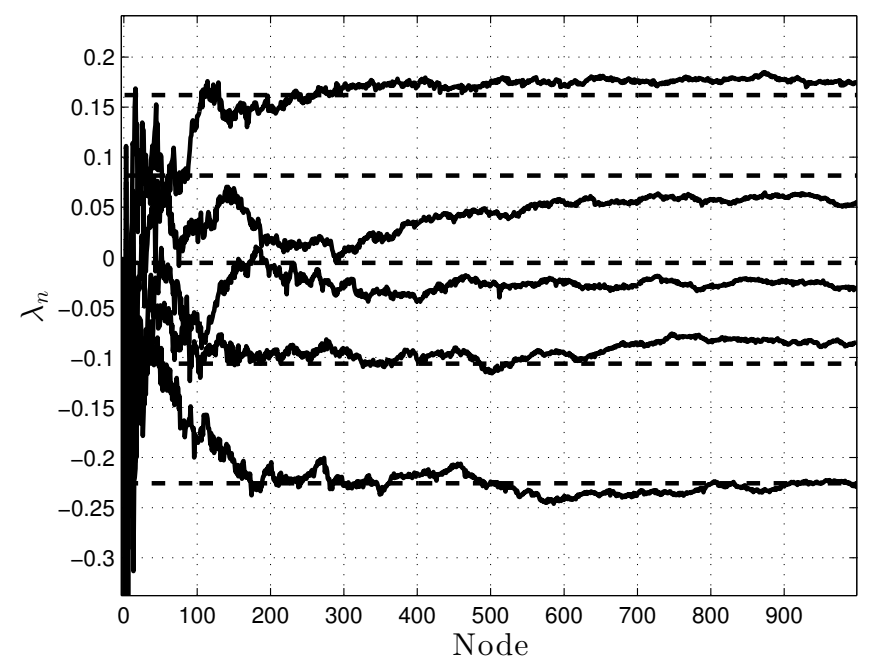

Fig. 3. Figure showing an instantaneous realization of the network's finite Lyapunov exponent (30) as a function of the number of nodes in the network. The relays are assumed to apply a unit gain and the channel coefficients between each node are assumed to be i.i.d. From lower curve to upper curve, $\mathbb{E}\left[\left|g_{i} h_{i j}\right|^{2}\right]=0.25,0.2875,0.325,0.3675,0.4$.

lines). The five capacity plots are obtained by considering $\mathbb{E}\left[\left|g_{i} h_{i j}\right|^{2}\right]=0.25,0.2875,0.325,0.3675,0.4$. From the figure, it can be clearly seen that if $\mathbb{E}\left[\left|g_{i} h_{i j}\right|^{2}\right]<0.35$, the endto-end capacity appears to decay exponentially across the network. As mentioned above, $\mathbb{E}\left[\left|g_{i} h_{i j}\right|^{2}\right]<0.35$ corresponds to the region in which $\lambda<0$. Furthermore, the capacity curves appear to trend along the line $\exp \left(n \lambda_{\gamma}\right)$, as predicted by (23). Consequently, we find that our numerical results our in complete agreement with the theory presented in Theorem 1. Specifically, positive Lyapunov exponents imply no capacity

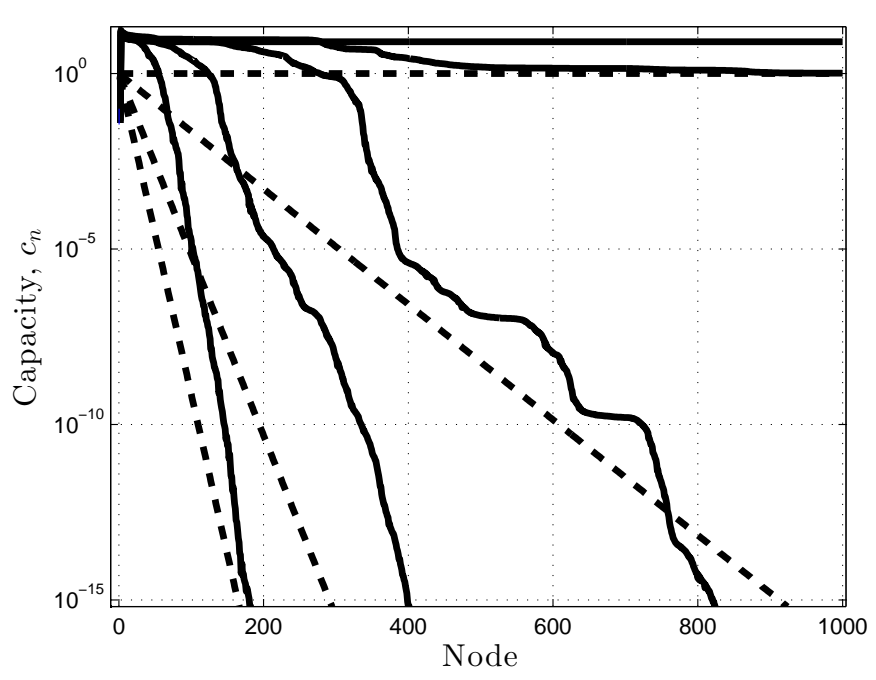

Fig. 4. Figure showing capacity (dashed lines) (22) as a function of number of nodes in network. Solid lines show $\exp \left(n \lambda_{\gamma}\right)$. The relays are assumed to apply a unit gain and the channel coefficients between each node are assumed to be i.i.d. From lower curve to upper curve, $\mathbb{E}\left[\left|g_{i} h_{i j}\right|^{2}\right]=$ $0.25,0.2875,0.325,0.3675,0.4$.

decay, while negative Lyapunov exponents imply that the capacity will decay according to $\exp \left(n \lambda_{\gamma}\right)$. As with before, it is not until the network becomes sufficiently large $(n \approx 100)$ that the trends begin to emerge, and for smaller $n$ we find that capacity decay tends to be less than that predicted by the corresponding dashed lines. It is important to note that the upper two curves of this figure will correspond to exponential power growth across the network. This will be shown in the next figure (Fig. 5), discussed in the next paragraph.

With reference to Fig. 5, we will now demonstrate Lemma 1. This figure is of a similar structure to the previous figure, but now the solid lines represent power as a function of network size, and the dashed lines show $\exp (n \max \{0,2 \lambda\})$ (see (27)). As with the previous figure, the five power curves are obtained by considering $\mathbb{E}\left[\left|g_{i} h_{i j}\right|^{2}\right]=0.25,0.2875,0.325,0.3675,0.4$. From this figure, we find that when $\mathbb{E}\left[\left|g_{i} h_{i j}\right|^{2}\right]>0.35$ the transmit power at the $n$th node grows exponentially. Furthermore, the transmit power appears to trend along the dashed line $\exp \left(n \lambda_{X}\right)$, as predicted by (27). Consequently, again we find that our numerical results our in complete agreement with the theory presented in Lemma 1 . Concretely, $\lambda<0$ implies no exponential power growth, while $\lambda>0$ implies that the power will trend along $\exp \left(n \lambda_{X}\right)$.

The practical implications of our theoretical analysis are that AF relays may be effectively deployed within delay intolerant linear networks. Such networks may arise when communicating along, e.g., vehicular convoys or railway line sensors, etc. Furthermore, our theoretical results provide design guidelines that should be followed when constructing such networks. In particular, these networks should be constructed so that their Lyapunov exponent is zero, which is achieved by appropriately 


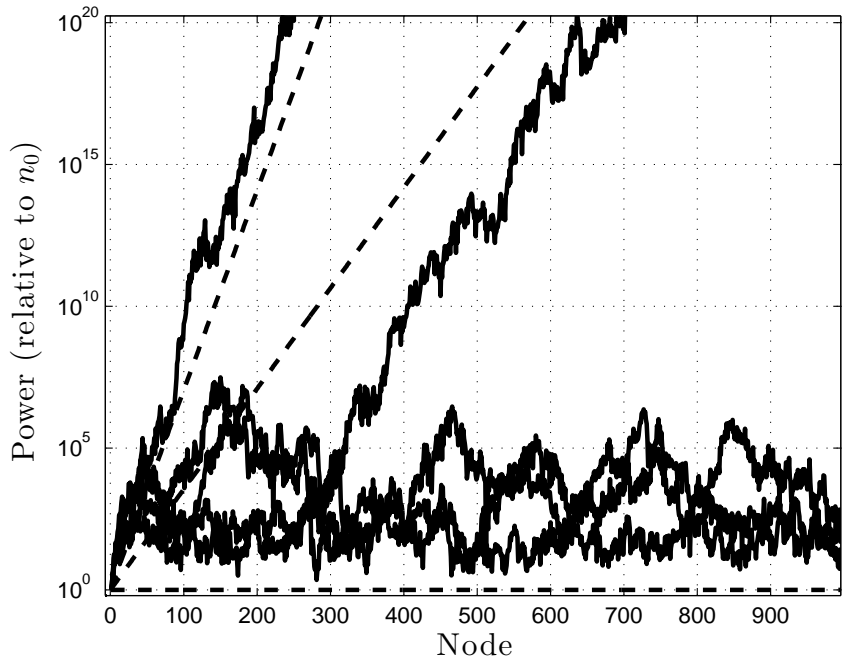

Fig. 5. Figure showing transmit power (solid lines) (21) as a function of number of nodes in network. Dashed lines show $\exp \left(n \lambda_{\gamma}\right)$. The relays are assumed to apply a unit gain and the channel coefficients between each node are assumed to be i.i.d. From lower curve to upper curve, $\mathbb{E}\left[\left|g_{i} h_{i j}\right|^{2}\right]=$ $0.25,0.2875,0.325,0.3675,0.4$

selecting the relays' amplification factors. This will allow networks to be constructed for which exponential capacity decay and exponential transmit power growth are simultaneously avoided across the network.

\section{CONCLUSION}

In this paper, we have motivated the study of continuous random Fibonacci sequences. In particular, we have shown that the Lyapunov exponent of these sequences can be used to establish capacity and power scaling laws for cooperative $\mathrm{AF}$ relay networks. Furthermore, with the laws that we have established, we are able to see that cooperative AF networks can be constructed that avoid both exponential capacity decay and exponential transmit power growth across the network. This is achieved by ensuring that the Lyapunov exponent of the sequence is zero. Our results will have practical implications to the design of delay intolerant linear networks such as, e.g., vehicular convoys or railway line sensors, etc. To conclude the paper, we provided some numerical examples to demonstrate our theory. Future work should focus on calculating explicit expressions for the Lyapunov exponent associated with random Fibonacci sequences.

\section{REFERENCES}

[1] D. E. Simmons, J. P. Coon, and N. Warsi, "Capacity and Power Scaling Laws for Finite Antenna MIMO Amplify-and-Forward Relay Networks," IEEE Transactions on Information Theory, vol. 62, pp. 1993-2008, April 2016.

[2] D. Simmons, J. P. Coon, and N. Warsi, "Capacity and Power Scaling Laws for Finite Antenna Amplify-and-forward Relay Networks," in 2016 IEEE International Symposium on Information Theory (ISIT), pp. 18591863, July 2016.

[3] J. Laneman, D. Tse, and G. Wornell, "Cooperative Diversity in Wireless Networks: Efficient Protocols and Outage Behavior," Information Theory, IEEE Transactions on, vol. 50, pp. 3062 - 3080, dec. 2004.
[4] T. Cover and A. E. Gamal, "Capacity Theorems for the Relay Channel," IEEE Transactions on Information Theory, vol. 25, no. 5, pp. 572-584, 1979.

[5] A. M. Tulino and S. Verdú, Random Matrix Theory and Wireless Communications, vol. 1. Now Publishers Inc, 2004.

[6] L. Arnold, Random Dynamical Systems. Monographs in Mathematics, Springer, 1998.

[7] J. Wagner and A. Wittneben, "On Capacity Scaling of Multi-Antenna Multi-Hop Networks: The Significance of the Relaying Strategy in the "Long Network Limit";", Information Theory, IEEE Transactions on, vol. 58, pp. 2107-2133, April 2012.

[8] S.-p. Yeh and O. Lévêque, "Asymptotic Capacity of Multi-Level Amplify-and-Forward Relay Networks," in Proceedings of the 2007 IEEE International Symposium on Information Theory, no. LTHICONF-2006-011, 2007.

[9] L. Fibonacci and L. Sigler, Fibonacci's Liber Abaci: a Translation into Modern English of Leonardo Pisano's Book of Calculation. Springer Science \& Business Media, 2003.

[10] S. Douady and Y. Couder, "Phyllotaxis as a Dynamical Self Organizing Process Part I: the Spiral Modes Resulting from Time-periodic Iterations," Journal of theoretical biology, vol. 178, no. 3, pp. 255-273, 1996.

[11] S. Basin, "The Fibonacci Sequence as it Appears in Nature," Fibonacci Quarterly, vol. 1, no. 1, pp. 53-56, 1963.

[12] M. Livio, The Golden Ratio: The Story of Phi, the World's Most Astonishing Number. Broadway Books, 2008.

[13] D. Viswanath, "Random Fibonacci Sequences and the Number 1.13198824...," Mathematics of Computation of the American Mathematical Society, vol. 69, no. 231, pp. 1131-1155, 2000.

[14] M. Embree and L. N. Trefethen, "Growth and Decay of Random Fibonacci Sequences," in Proceedings of the Royal Society of London A: Mathematical, Physical and Engineering Sciences, vol. 455, pp. 24712485, The Royal Society, 1999.

[15] É. Janvresse, B. Rittaud, and T. De La Rue, "How do random Fibonacci sequences grow?," Probability Theory and Related Fields, vol. 142, no. 3-4, pp. 619-648, 2008.

[16] E. Makover and J. McGowan, "An Elementary Proof that Random Fibonacci Sequences Grow Exponentially," Journal of Number Theory, vol. 121 , no. 1, pp. 40-44, 2006.

[17] P. L. Krapivsky et al., "Random Fibonacci Sequences," Journal of Physics A: Mathematical and General, vol. 34, no. 42, p. 9065, 2001.

[18] B. Rittaud, "On the Average Growth of Random Fibonacci Sequences," Journal of Integer Sequences, vol. 10, no. 2, p. 3, 2007.

[19] T. M. Cover and J. A. Thomas, Elements of Information Theory. John Wiley \& Sons, 2012 